Reichmann, Oskar (1989): Frühneuhochdeutsches Wörterbuch, Bd. 1, Berlin/New York.

Roelcke, Thorsten (1998): Die Periodisierung der deutschen Sprachgeschichte, in: Besch, Werner/Betten, Anne/Reichmann, Oskar/Sonderegger Stefan ( $\mathrm{Hg}$ ) Sprachgeschichte. Ein Handbuch zur Geschichre der deutschen Sprache und ihrer Erforschung, 2, vollstandig neu bearb. und erw, Aufl., Telbd. 1 (Handbücher zur Sprach- und Kommunikarionswissenschaft, 2.1), Bedin/New York, 798-815.

Scheren, Wilhelm (1875): Geschichte der deutschen Dichtung im elfren und zwölfen Jahthundert (Quellen und Forschungen zur Sprach- und Culturgeschichte der gemanischen Vöker, 12), Straßburg/London.

Scherer, Wilhelm (1878): Zur Geschichte der Deutschen Sprache, 2. Ausg., Berlin [zitert nach: Neuer Abdruck, 1890].

Scherer, Wilhelm (1883): Geschichte der Deutschen Litteratur, Berlin.

Schwab, Ute (1991): Tötende Töne. Zur Fiedelmetaphorik im Nibelungenlied, in: Geist und Zeir. Wirkungen des Mitrelalters in Lirerarur und Sprache. Festschrift für Roswitha Wisniewski zu ihrem 65. Geburtstag, Frankfurt a.M. u.a., 77-122.

Splett, Jochen (1987): Das Wortschatzargument im Rahmen der Gattungsproblematik des Nibelungenliedes, in: Knapp, Fritz Peter (Hg): Nibelungenlied und Klage. Sage und Geschichte, Struktur und Gattung. Passauer Nibelungengespräche 1985. Heidelberg, 107-123.

Thim-Marbrey Christiane (1990): Atributives Partizip Präsens im Mittelhochdeutschen, in: PBB 112, 371-403.

Wegera, Klaus-Peter (1987): Grammatik des Frühneuhochdeutschen. Beiträge zur Laut- und Formenlehre, hg. von Hugo Moser, Hugo Stopp und Werner Besch, Bd. 3, Heidelberg.

Wegera, Klaus Peter (2000): Grundlagenprobleme einet mittelhochdeutschen Grammark, in: Besch, Werner/Betten, Anne/Reichmann, Oskar/Sonderegger Stefan (Hg): Sprachgeschichte. Ein Handbuch zur Geschichte der deutschen Sprache und ihrer Erforschung, 2., vollständig neu bearb. und erw. Aufl., Teilbd. 2 (Handbücher zur Sprach-und Kommunikationswissenschaf, 2.2), Berlin/New York, 1304-1320.

Wiessner, Edmund/Burger, Harald (1974): Die höfsche Blitezeit, in: Maurer, Friedrich/Rupo, Heinz ( $\mathrm{Hg}$ ): Deutsche Wortgeschichte, Bd. 1, 3., neubearb. Auft (GrundiB der germanischen Philologie, 17/I), Berlin/New York, 187-253.
Damaris Nübling (Mainz)/Renata Szczepaniak (Hamburg)

\section{Was erklärt die Diachronie für die Synchronic der deutschen Gegenwartssprache? Am Beispiel schwankender Fugenelemente*}

\section{Zweifelsfälle im Allgemeinen}

Zu dem Strauß der Bindstrichlinguistiken gesellt sich derzeit ein weiteres, besonders interessantes und vielversprechendes Exemplar, die sog. Zweifelsfal-Linguistik. Thre Entstehung kann man mit dem Linguistik online-Heft ,Sprachliche Zweifelsfille. Theorie und Empirie" (herausgegeben von Wolf Peter Klein) auf das Jahr 2003 datieren. Hier wurde den Zweifelsfallen, die bisher eher Gegenstand der Sprachberatung waren, die linguistische Weihe verliehen, d.h. sie werden seither als "linguistischer Gegenstand" (so der Titel der Einleitung) mit theoretischem Anspruch gesehen ( $s$. hierzu auch Klein 2009 mit dem Titel "Zweifelsfalle als Herausforderung fur die Sprachwissenschaf ${ }^{\text {") }}$. In der erwähnten Einleitung von Klein (2003) erfolgt die Definition des sprachlichen $Z$ weifelsfalls:

Ein sprachlicher Zweifelsfall ist eine sprachliche Einheit (Wort/Wortform/Sarz), bei der ko mpetente Sprecher (a.) im Blick auf (mindestens) zwei Varianten $(a, b .$.$) in Z$ weifel getaten (b.) können, welche der beiden Formen (standardsprachlich) (c) korrekt ist (vgl. Sprachschwankung, Doppelform, Dublette). Die beiden Varianten eines Zweifelsfalls sind form seitig of teilidentisch (d) (z B. dubiosldubiös, bosharlioslich des Automat (sic) des Automaten, Rad fabrentradfahren, Stavb gesaugt/staubgesaugt/gestaubsaugt). (Klein 2003, 2; Spertungen im Original).

Die gesperten Begriffe werden als die wesentichen Bedingungen fur einen Zweifelsfall gewertet: (a.) Kompetente Sprecher geraten in Zweifel, nicht Sprachwissenschafterinnen und auch nicht (Erst-/Zweit-) Spracherwerbermnen. Auch muss der Zweifel in (b.) metasprachliches Bewusstsein

"Dieser Beitrag ist im Rahmen des Mainzer Forschungsprojekts, Determinanten sprachlicher Variation" entstanden "

Gemeint ist wohl Automats. 
enkennen lassen. Weiter ist der Zweifelsfall (c.) in der Standardsprache zu verorten (wie immer diese zu fassen sein mag, zumindest muss sie intendiert sein). Mit der (d.) formseitigen Teilidentität ist v.a. die Grammatik angesprochen, die im Zweifelsfall nur minimal unterschiedliche Varianten generiert. Klein $(2003$, 4) nennt hierfür Beispiele: „Stellt Friede oder Frieden dic korrekte Worform dar? Gehört in das Kompositum Kriegfihhrung ein Fugen-s? Muss im Genitiv Kindes oder Kinds gesagt werden?"

Aus historisch-linguistischer Perspektive handelt es sich sehr häufig um Fälle sich gegenwärtig vollziehenden Sprachwandels, d.h. was heute an seismischen Bewegungen registriert wird, hat seinen Herd, um in diesem Bild zu bleiben, oft im Frühneuhochdeutschen oder noch früher. Im universitären sprachgeschichtlichen Unterricht erreicht man mit dieser Sichtweise auch die letzten an der Nuitzlichkeit der Sprachgeschichte Zweifeinden (es sind allerdings viel weniger, als man glauben möchte), d.h. Seminare mit diesem Thema muss man in der Regel teilen, da der Andrang zu groß ist. Gerade für die zukünftigen LehrerInnen ist es wichtig, von der tichtig/falsch-Zentriertheit von Zweifelsfillen wegzukommen und starrdessen der Ratio dieses Phänomens näherzukommen (um dann bessere Anleitungen geben zu können). In Veranstaltungen zu Zweifelsfälen clangt man übrigens eine beträchtliche diachrone Tiefe, d.h. die Bereitschaft, sich in das Problem, seine Genese und seinen Hintergründe einzuarheiten, ist erfreulich hoch. Interessant (und noch nicht erforscht) ist dabei die unterschiedliche Salienz grammatischer Zweifelsfalle: Während die Fugensetzung sofort als Zweifelsfall erkannt und bestätigt wird, ist es bei der schwankenden Flexion zweier koordinierter Adiektive im Dativ ohne Determinans (unter großem finanziellem:/finanziellen? Aufwand) anders. Auch wenn die Korpora die Schwankung zwischen Parallel- und WechsetHexion zweifelsfrei als Zweifelsfall ausweisen (ca. zwei Drittel Wechselflexion, ca. ein Drittel Parallelflexion), so erreicht diese Flexionsunsicheheit keinen hohen Bewusstheitsgrad. Die höchste Salienz erreichen ubrigens orthographische Zweifelsfälle (wie die im Zitat genannten Getrennt-/Zusammenschreibungen), danach Wortbildungsprobleme wie die (Un) Trennbarkeit von Präfixen vom Typ gedownloadetdowngeloadet.

Was die Kenntnis der Sprachgeschichte für solche synchronen Zweifelsfalle neben ihrer Exklärung leistet, ist die Angabe der Richtung des Wandels: Was gegenwärtig in beide Richtungen gleichermaßen ausschlagen kann, erweist sich aus diachroner Perspektive langfristig als ein Übergang von $a>b$

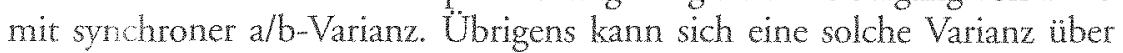
Jahrhunderte erstrecken, was ein Blick in die Sprachberatungsbücher des 19. und 20. Jhs. bzgl. der Parallel-/Wechselfexion erweist (z.B. Wustmann 1891. Matthias 1906. Andresen 1923, Steche 1925 und 1927). Auch die Grammariken dieser Zeir behandeln diese Fälle oft sogar sehr ausfuihrlich.
Wie Klein (2009) ausführt, gilt es mehrere Faktoren für die Entstehung bzw. Existenz von Zweifelsfällen zu unterscheiden: Die Schriftsprachlichkeit, die Varietätenvielfalt (je mehr Varietäten, desto mehr Zweifelsfälle) sowie der Grad des metasprachlichen Bewusstseins in einer Kultur (das durch die Schriftkultur wiederum gefördert wird). Einige dieser Faktoren sind bekanntlich auch für Sprachwandel verantwortlich, allen voran die Varietätenvielfalt (interner Sprachkontakt).

Im Folgenden soll anhand des Zweifelsfalls der Fugensetzung gezeigt werden, in welche Tiefen man mit der konsequenten Frage nach dem Warum gelangt - und wie schnell man auch erkennen muss, dass es an Forschung dazu fehit.

Da Zweifelsfalle, wie erwähnt, standardsprachlich vorkommen müssen, lassen sie sich auch relativ einfach in standardsprachlichen Korpora wie Cosmas II des IDS nachweisen, meist sogar - je nach Größe des Korpus - zahlreich, was die Suche nach ihren Determinanten u.U. erleichtert (erwa bei der Frage nach der Bedingtheit von Parallel- vs. Wechselflexion bei zwei koordinierten Adjektiven im Dativ). Diese Nachweisbarkeit in Datenbanken gehört zu den Identifikationsmöglichkeiten von Zweifelfällen, wie sie in Klein $(2003,6 f f$.) genannt werden. Dazu gehören auch Erfahrungsberichte von Sprachberatungsstellen. An diesen lassen sich gut die unterschiedlichen Salienzgrade von Zweifelsfallen ablesen.

In Bezug auf Zweifelsfalle morphologischer Art lassen sich derzeit die folgenden 10-15 größeren "Baustellen" identifizieren:

- Silbische vs. unsilbische starke Genitivendung: des Buch $\left.(e)^{2}\right)^{2}$

- Genitivendung vs. Null: des Iran(s?), des Kosovo(s?), des Präteritum(s?),

- Partitiver Genitiv: eine Tasse frischgebratuten Kaffees?/.. frischgebrauter Kaffee?

- Genitiv-/Dativ-Rektionsschwankungen bei Präpositionen (in beide Richtungen): gemäß dem?/des? Autor(s); während des?'dem? Spiell(s);

- Fugenelemente: Schaden(s?)ersatz, Interessen(s?)bekundung, Respekt(s?) person:

- Starke vs. schwache Adjektivflexion meist bei Zeitangaben: Ende dieses?/diesen? Monats;

- Starke vs. schwache Adjektivflexion bei Koordination ohne Artikel. wort: unter großem finanziellem(?)/fnanziellen(?) Aufwand;

- Flexion von Deadjektiva nach Personalpronomen: wir Deutsche?/wir Deutschen? wir Arbeitslose?/wir Arbeitslosen?

2 Was die Varianz der starken Genitrvendung -es/-s betriff, so liegt mit Szczepaniak (im Druck a) die erste korpusbasierte Untersuchung dieses phänomens vor. 
- Substantivflexion: des Eisbaren/Eisbärs? des Autors/Autoren? Pluralschwankungen vom Typ die Pizzas/Pizzen, die Wagen/Wagen, die Worte/Wörter, die LKWILKW' etc.

- Verbalmorphologie: Hier sind vielfaltige stark/schwach-Schwankungen in verschiedenen Stadien erkennbar, z.B. beim Imperativ (ess//iss!), in der Wechselfexion (du fragst/fragst), im Präteritum (er buklbackte), im Konjunktiv (sie stindelstïnde, gewönnelgewänne), im Partizip Perfekt (gesät/gegoren, aber auch angewandtangewendet);

- Adiektivkomparation: krummer/krïmmeri, kranker/kränker?

- Snmerlative komplexer Adjektive: das beachtetste? meist beachtete? Buch;

- Trannbarkeir von Partikelverben: downgeloadet?/gedownloadet?

- Fugenelemente innerhalb von Komposita: Seminar(s?)arbeit, Hauptseminar(s?) arbeit, Referat(s?)besprechung, Präteritum(s?) schund; dieser Zweifelsfall soll im Folgenden beleuchter werden.

\section{Der Zweifelsfall im Besonderen: Schwankende Fugenelemente}

2.1 Fugenschwankungen im

Wörterbuch der sprachlichen Zweifelsfalle" (Duden Band 9)

Das ausfuhrlichste, aus langïhriger Sprachberatung schöpfende Verzeichnis sprachlicher Zweifelsfalle ist der Duden-Band 9 "Richtiges und gutes Deutsch - Wörterbuch der sprachlichen Zweifelsfalle" (2007 in der 6. Aulage). Selbstverständlich besteht sein primäres Ziel darin, Handlungsanleitungen zu geben und weniger dem Phänomen linguistisch oder gar sprachhistorisch auf den Grund zu gehen. Genau dies sollte Gegenstand der Disziplin einer Zweifelsfall-Linguistik sein, wie diese von Klein (2003; 2009) initiert wurde.

Dennoch gehen wir zunächst von der Darstellung dieses Zweifelsfalls im "Wörterbuch der sprachlichen Zweifelsfalle" (2007) aus, um zu sehen, was hier als problematisch dargestell wird. Neben den konkreten Zwei felsfallen (z.B. Schiff-/Schiffs-als schwankendes Erstglied etwa in Schiff(s). baw oder Schweine-Schweins-in Schwein(e?/s?)braten) werden auch die von Schwankungen betroffenen Themen lemmatisiert. Handelt es sich dabei um sehr häufg nachgefragte Komplexe, dann wird zu dem Thema ein ganzer, of mehrseiriger Uberblicksartikel eingestell. Wenn man also dem Thema "Fugensetzung" nachgehen will, findet man unter "F-" sowohl den Eintrag "Fugen-s", dem ein dreiseitiger Uberblicksartikel gewidmet ist, als auch "Fugenzeichen", dessen Bedeurung in dem berreffenden Lemmaartikel abgehandelt wird, also weniger exponiert wird.
Genau diese Hierarchie entspricht auch unseren Beobachrungen baw. den Sammlungen von Fugenschwankungen, ${ }^{3}$ die deutlich machen, dass es praktisch nur ein Fugenelement gibt, das in Schwankungen involviert ist, nämlich das Fugen-s. Der Eintrag zu "Fugenzeichen" erlautert dagegen nur den grammatischen Status von Fugenelementen, nämlich Komposita zu gliedern, aber keine inhaldiche Funkrion zu tragen. Außserdem wird die Entstehung von Fugenzeichen aus fruheren Flexionselementen (Genitivkonstruktionen) sowie thre spätere analogische Ausdehnung beschrieben, hier am Beispiel von Bischofsmütze, das tatsächlich auf des Bischof Mütze zurückgehen könnte, im Gegensatz zu Bischofskonferenz, wo dies auszuschließen ist, da sich hier eine potentielle Genitivlesart verbietet. Schließ lich erfolgt ein Verweis auf den Artikel "Fugen-s".

Dieser Uberblicksartikel ist dreigeteilt: 1. Komposita mit Fugen-s, 2. Komposita ohne Fugen-s, 3. Komposita mit schwankendem Gebrauch des Fugen-s. Im Allgemeinen nehmen Fugen $-s$ "Komposita mit bestimmten Erstgliedern" wie z.B. Armut, Bahnhof, Liebe, Hilfe, Geschichte, dann substantivierte Infinitive als Erstglied ( $\mathrm{z} . \mathrm{B}$. Verbrechensbekämpfung) und schließlich Erstglieder mit den Ableitungssuffixen -tum, -ing, -ling, -heit, -keit, schaft, -ung, -ion, -ität, -at und -um. Ohne Fugen-s stehen in der Regel einsibige feminine Erstglieder oder solche auf $-\epsilon,-u r,-i k$, schwache Maskulina als Erstglieder, Erstglieder auf -er und -el sowie solche auf -[s], -[]] oder -[st].

Als schwankend, aber gleichermaßen berechtigt ausgewiesen werden Komposita mit -steuer (z.B. Körperschaff(s) steuer) und -straße (z.B.

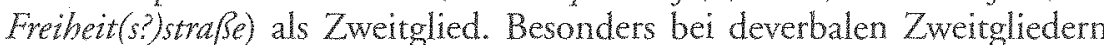
(Verbalabstrakta, Partizipien) werden Schwankungen erwähnt, wobei wegen des Objektcharakters des Erstglieds keine Fugensetzung empfohlen wird: "Hilfelestung, Kriegfibrung (aber: Kriegserklanng)"; "verfassunggebend (seltener: verfossungsgebend); (substantivier): Vertragshließknde; Gewerbetreibende. Ausnahmen kommen vor (kriegsentscheidend, stantser. haltend)" (337). Schlieblich nehmen "häufg " mehrgliedrige Komposita in der sog. "Hauptuge" ein Fugen-s" "So heiBt es Friedhofstor gegenüber der zweigliedrigen Bildung Hoftor, Mittemachtsstunde gegenuber Nachistunde" (337). Ein letzter Absatz betriff die Bindestrichschreibung und erlaubr

3 Für die Versorgung mit Fugenschwankungen danken wis heralich Yvonne Goldammer Franziska Münzberg und Melanie Kunkel von der Duden-Sprachberatung.

4 Interessanterweise wird hier mit unverfuotem Krieg führung ein gerade nicht schwankendes Kompositum dokumentiert, während Klein (2003) genau dasselbe Kompositum Krieg(s) fuihrung - als Beispiel für das Gegenteil, eine Fugenschwankung, anfïhrt. Tarsächlich handelt es sich um einen geradezu klassischen Zweifelsfall, der sich einer informellen Googlerecherche (rom 4.1.2010) we folgt verhält: Krieg $+5+$ fithrang. 251.000 Krieg + Q fithrung: 142.000 , d.h. $64 \%: 36 \%$ 
sie nach einem Fugen-s nur in Ausnahmefällen waus Gründen der Übersichtichkeit" (337) (〈Stadtverwaltungsoberinspektorin/StadtverwaltungsOberinspektorin $\rangle$ ). Soweit die Duden-Empfehlungen.

\subsection{Zweifelsfälle der s-Verfugung}

Eine hier ansetzende Zweifelsfall(s?) linguistik betrachtet die typischen Schwankungsfalle als die Spitze des Eisbergs, versucht dabei aber, den unsichtbaren, viel größeren Eisberg auch in den Blick zu nehmen und die Regularitäten hinter den Schwankungen sichtbar zu machen. Eine diachrone Linguistik geht noch eine Dimension weiter und ist an dem dahinterliegenden Sprachwandel interessiert, wenn es sich denn um einen solchen handelt (und nicht nur um einen synchronen Regelkonflikt). Insgesamt ist $\mathrm{u} . \mathrm{E}$. gerade in Hinblick auf die Fugenschwankungen der folgenden Ermahnung von Wegener $(2005,157)$ zuzustimmen:

Die Fugenelemente sind ein Paradebeispiel dafür, dass man manchmal ohne historisches Wissen, ohne Kenntnis fruherer Sprachstufen, nicht auskommt und zu holnungslosen Fehldeutungen verleitet wird.

Tabelle 1 listet einige besonders virulente und nach Ausweis verschiedener Korpora stark schwankende Beispiele auf, wobei wir uns hier nur auf $N+N-K o m p o s i t a$ beschränken. Doch scheinen sich Komposita mit adjektivischen Zweitgliedern ebenso zu verhalten. Insgesamt düfte die Zahl fugenschwankender Komposita in die Hunderte gehen, doch wurden sie bis dato noch nicht erschöpfend erfasst. ${ }^{5}$
Abitur(s)feier

Antrag ( $(?)$ formular

Interessen(s?)vertretung

Präteritum(s?)schwund

Seminar(s?)arbeit

Referat(s)besprechung

Subjek(s?)pronomen

\begin{tabular}{|c|c|}
\hline Schaden(s?)ersatz & Datum(s?)angabe \\
\hline Mehrwert(s)srener & Denkmal(s?)plege \\
\hline Erbschaft(s) steuer & Gewriche(s?)heber \\
\hline Krieg(s?)fuhrung & Stellung(s?)nahme \\
\hline Dreieck(s?)tuch & Lehramt(s?)kandidat \\
\hline Arbeit(s?)nehmer & Respekt(s?)person \\
\hline Schiffahrt(s3)unfall & Widerufrecht(s?)belchrung \\
\hline
\end{tabular}

Tab. 1: Einige Zweifelsfalle der $s$-Verfugung
5 Alsichtlich haben wir auf solche (häufig vorkommenden) Zweifelsfalle verzichtet, deren Zweitglied mit einem $s[z]$ beginat (wie Advent(s?)singen, Antrieb(s?) system), un ausraschließen, dass es sich nur um graphematische Umsetzungsprobleme eines akustischen problems handelt.
Grau hinterlegt wurde ein besonderer, bereits erwähnter Fall von Fugenschwankung, der diaphasisch bedingt zu sein scheint (und den wir im Folgenden ausblenden): Es kommt immer wieder vor, dass Komposita fachsprachlich unverfugt bleiben, während sie gesprochen- und umgangssprachlich verfugt werden. Dazu gehören die vom Duden Band 9 hervorgehobenen Komposita mit -steuer als Zweitglied, es gibt aber noch mehr solche Fälle aus der juristischen Fachsprache, z.B. Schaden(s?)ersatz, Sorgerecht(s?) prozess, Sachstand(s?) anfruge. Aus der Linguistik kennt man Fälle wie Namenkunde oder Präteritumschwund, die außerhalb dieser Disziplin fast immer zu Namenskunde, Präteritumsschwund verfugt werden. Zum Fall Schiff(s:) bau schreibt Duden Band 9: "Schifflau wird besonders im Ingenieurwesen ohne Fugen-s gebraucht; daneben kommt auch Schiffsbau vor" (793).

Bei der Frage nach der Richtung des Wandels geben auch diese Fülle Auskunft, wenn man die fachsprachliche Version, was sinnvoll ist, als die konservativere Form betrachtet: Der Weg fuhrt eindeutig von der Nullzu s-Fuge.

Was die Null- oder Nichtverfugung betriffe, so ist diese als der unmarkierte Normalfall zu betrachten: Ca. $58 \%$ aller Komposita werden nach Kürschner (2003), der ein Zeitungskorpus untersucht hat, nicht verfugt (bei Erzählprosa kommen Ortner at al. 1991 sogar auf 72,8\%). Es folgt dann schon mit $25 \%$ die $s$-Fuge und mit $11 \%$ die (e) n-Fuge. Die restlichen $6 \%$ verteilen sich auf die er-, die es-, die - $(e) n s$ und die $e$-Fuge. $\mathrm{Da}$ diese $6 \%$ unprodultiv sind, vernachlässigen wir sie im Folgenden (zu Näherem s. Augst 1975, Fuhrhop 1996, 1998, Eisenberg 2006, Nübling/Szczepaniak 2008, 2009). Relativ einfach lässt sich das Verhalten der (e) $n$-Fuge beschreiben, die fast nur schwache Substantivllassen betrifft und dabei dem Erhalt bzw. der Erzeugung von Trochäen dient: An einsilbige bzw. finalbetonte Erstglieder tritt silbisches -en-, an zweisilbige nichtsilbisches -n-(Fráu+en+forschung, Herrtentschokolade, Soldat+en+treffen vs. Dáme+n+abteilung, Künde+n+dienst). ${ }^{6}$ Das Fugenelement besteht dabei genau in dem Material, das zur Nom.Sg.-Form hinzutritt, wenn das betreffende Nomen als Bestimmungswort in ein Kompositum eingeht. Dabei kommt es nicht selten dazu, dass ein und dasselbe Nomen unterschiedliche Fugenelemente nehmen kann, je nach dem folgenden Grundwort, vgl. Kind $\rightarrow$ Kind + er+wagen, Kindtes+wohl Kind $+\emptyset+b e t$. Kind $+s+k o p f$. Wie an diesem Beispiel auch deutlich wird, alterniert die s-Fuge nicht in der Weise mit der es-Fuge, wie dies die n-mit der en-Fuge tut: Dies s-Fuge steht oft auch nach Einsilbern, d.h. die Trochäenerzeugung

6 Ab jetzl folgen wir der Konvention, die Fugenelemente durch „, " vom Stamm abzutrennen. 
ist nicht das leitende Prinzip. Da diese Elemente nicht miteinander austauschbar, sondern fest distribuiert sind (*Kind+es+kopf. *Kind+er+wohl, *Kind+s+wagen), handelt es sich auch nicht um Zweifelsfalle.

2.3 Der lange Weg der s-Fuge von der Syntax bzw. der Flexion in die Wortbildung ...

Bei den swerfugten Komposita lässt sich der Weg von einer einstigen Genitivphrase (a) ubber die Reanalyse (b) zu einem (Genitiv-)Kompositum (c) nach Demske (2001) wie folgt skizzieren:?

a) [[debet Bischofs $\left.\mathrm{N}_{\mathrm{N}}\right]_{\mathrm{NP}}$ Mütze $\left.]_{\mathrm{NP}}\right\rangle$

$$
\text { b) } \left.\left[\mathrm{des}_{\mathrm{Det}}[\text { Bischofs Mürze }]_{\mathrm{N}}\right]_{\mathrm{N}\}^{3}}\right\rangle
$$

c) $\left[\text { die }_{\text {Det }}[\text { Bischofsmütze }]_{N}\right]_{N P}$

Diese Lexikalisierung von Phrasenstrukturen findet im Fruhnhd. statt (s auch avlov 1983). Dabei geht die Lesart der ersten (Genitiv-) Konstituente von einer referenziellen in eine generische über (Demske 2001) Dieser neue Genitiv-Kompositionstyp, der ein neues Wortbildungsmuster eröfnet, wird in der Literatur, zurückgehend auf Jacob Grimm, als „uneigentiches Kompositum" bezeichnet (s. hierzu auch Nitta 1987, Sattler 1992. Solms 1999). Mit der Kongruenz des Artikels mit dem Kern- bzw. Kopfromen ist der Ubergang zum Kompositum vollzogen: Der Artikel wechselt vom Gen.Mask. des (Bischofs) zum Nom.Fem. die (Mütze). Das anzusezzende Zwischenstadium (b), in dem sich die Struktur von (a) aufzulösen beginnt, lässt sich dabei nur erschließen, nicht belegen (Groß-1 Klein-sowic Zusammen-/Getrenntschreibungen sind zu fruhnhd. Zeit wegen ihrer starken Variabilitat kaum als Indikatoren zu werten, s. hierzu Wegera/Plett 2000, 1597).

Moglicherweise bilden hier solche Phrasen die Brückenfunktion, die mehrdeutig sind, d.h. in denen der Artikel formal gleich bleibt wie etwa be einem femininen Genitiv (der) und einem maskulinen Nominativ (der). Allerdings funktioniert dies nicht mit dem Genitiv-s, da sich dieses (bis beute) nicht an Feminina heftet (von Eigennamen abgesehen), sondern nur mir dem Genitiy-(e)n oder, bei den starken Feminina, mit Null.

a) $\left[\text { der } \text { Sonnen }_{\mathrm{N}}\right]_{\mathrm{NP}} \operatorname{Tag}_{\mathrm{NP}}$

$$
\text { b) } \left.\left[\operatorname{der}_{\mathrm{Der}}[\text { Sonnen Tag }]_{\mathrm{N}}\right]_{\mathrm{NP}}\right\rangle
$$

c) $\left[\mathrm{der}_{\mathrm{Det}}[\text { Sonnentag }]_{\mathrm{N}}\right]_{\mathrm{NP}}$

Bzyt der sich im Fruhnhd, erst vollziehenden Großschteibung folgen wir dem nhd. Stand.
Auch im Genitiv Plural (der) wäre eine solche Brückenfunktion denkbar, allerdings wieder ohne das s-Flexiv:

a) $\left[\text { der }_{\text {Det }} \text { Pfaffen }_{\mathrm{N}}\right]_{\mathrm{NP}}$ Stand $\left.]_{\mathrm{NP}}\right\rangle$

$$
\text { b) } \left.\left[\text { der } r_{\text {Det }}[\text { Pfaffen Stand }]_{N}\right]_{N P}\right\rangle
$$

c) [der ${ }_{\text {Der }}$ [Pfaffenstand $\left.]_{\mathrm{N}}\right]_{\mathrm{NP}}$

Nur bei arrikellosen Phrasen vom Typ [[Feuers] Flammen] ) [Feuersflammen] kommt auch das s-Flexiv in Frage. - Um eine katalysatorische Wirkung dieser zweiten und dritten Rekonstruktion plausibel zu machen, müsste man korpusbasiert nachweisen, dass solche Konstruktionen mit gleichbleibender Artikelform denen mit wechselnder Artikelform zeitlich vorangehen. Dies ist bisher nicht geschehen. Insgesamt gilt, dass bis dato viel zu wenig diachrone Kompositions- und Fugenforschung geleistet wurde. Nach Demske (2001, 299) steigt „im 16., besonders aber im 17. Jh. [...] die Frequenz von Genitivkomposita sehr schnell an". Tatsächlich verwendet Luther noch kaum solche Genitivkomposita (Wilmanus 1899, Henzen 1965).

In einem zweiten, entscheidenden Schrit werden nun die morphologisierten s-Elemente produktiv, was sich zweifelsfrei erst dann nachweisen läst, wenn sie nicht mehr nur an starken Maskulina und Neutra haften, sondern auch an schwachen (Bauer+s+mann), wenn sie auf pluralisch zu interpretierende Erstglieder und vor allem wenn sie auf Feminina ubergehen. Ab hier verbietet es sich, noch von Genitivkomposita zu sprechen. So fallt die Bischof + stmütze durchaus unter den früheren Typ des Genitivkompositums, doch keinesfalls mehr die Bischoft-s+konferenz, der Freundtestkreis oder der Dreikönig+sttag, da die Erstglieder nur eine Purallesart exlauben. Auch im Fall singularischer Erstglieder verbietet sich of eine Genitivlesart: So ist ein Liebling+s+getrank keineswegs ein ,Getränk des Lieblings'. Massenhaft breiten sich indessen diese mitterwelle nur noch als Fugenelemente zu bezeichnenden Grenzmarker auf Feminina aus: Abfahnts+zeit, Geschicht+s+bewnsstsin, Weihnacht+s+gans, Betreung $+s+z e$ it etc. Regelmäßig verfugt werden, wie unter 2.1 bereits erwähnt, ausgerechnet feminine Derivationssuffixe wie -heit, - (ig)keit, -schaft. -ung, -ion, -itatt, und dies schon früh, d.h. bereits ab dem 16.17. Th. Wilmanns $(1899,531 \mathrm{f}$ ) dokumentiert für Autoren des 16. und 17. Ths Verfugungen wie Authoritäts+discurse, Religion + stvergleichung etc. Al diese aus ihren ursprünglichen Kontexten losgelösten s-Elemente sind sog. unorganische (Henzen 1965) oder unparadigmische Fugenelemente (Fuhrhop 1996, Eisenberg 2006). Sie sind in jedem Fall von der Flexionsmorphologie entkoppelt, ihre genus- und Aexionsklassengebundene Konditionierung haben sie abgebaut. Die Frage, die sich nun stell, ist, 
worin ihre Konditionierung heute besteht, d.h. wie die Fugengrammatik, uber die wir alle verfugen, beschaffen ist.

\section{4 und der Weg heute von der Wortbildung in die Phonologie:}

Das Fugen-s als rechter Wortrandverstäker

Dass der s-Fugenwandel heute noch in vollem Gang sein muss, belegen die vielen Zweifelsfalle. Die anderen Fugenelemente sind davon nicht annhernd so stark betroffen, d.h. sie tradieren eher ihre angestammten Distributionstegeln.

Per bisherige Stand der Fugenforschung bzgl. des Gros der Schwankungsalle bestand darin, Fugenelemente als Anzeiger morphologischer Komplexirät zu sehen: In dem Maße, in dem die morphologische Komplexitat des Erstglieds zunimmt, nimmt auch - grob gesagt - die Fugensetzung zu (Henzen 1965, Ortner et al. 1991, Fuhhop 1996; 1998, Gallmann 1998, Eisenberg 2006). Dies deutet auch das Duden-Wörterbuch der sprachlichen Zweifelsfille an, wenn es unter "mehrgliedrige Komposita" schreibt:

Hier wird häufg, aber nichr immer die Haupfuge durch das Fugen-s gekennzcichnet. So heibt es Friedhofstor gegenuber der zweigliedrigen Bildung Hoftor, Mitternachtsstande gegenuber Nachtstunde. Aber ohne Fugen-s: Fubballmeister, Kindbetteber u.a. (337)

Die Beispiele in Tabelle 2 legen ein solches Prinzip tatsächlich nahe: Es lassen sich sehr viele Beispiele für diesen Effekt finden. Erst Kürschner (2003) entdeckte in seiner korpusbasierten Untersuchung, dass nicht jegm liche morphologische Komplexität des Erstglieds zu vermehrter Fugensetzung tuhrt (s. Tabelle 3): Gerade dann, wenn das Erstglied seinerseits aus einem Kompositum besteht (und damit morphologisch besonders komplex ist), verhält sich die Fugenserzung wie bei jedem einfachen Kompositum, indem die Nullfuge dominiert (hier sogar noch stärker als im Durchschnit). Nur bei derivationell komplexen Extgliedern kehren sich die Verhälnisse um, d.h. hier wird zu $67,5 \%$ s-verfugt und nur zu $29 \%$ nulverfugt (s. Tabelle 3 ).

\begin{tabular}{|c|c|c|}
\hline Nr. & monomorphematisch & polymorphematisch \\
\hline 1 & Hoffor & Friedhof $+s+$ tor \\
\hline 2 & Marktbude & Jabrmatht $+s+b u d e$ \\
\hline 3 & Werkzeng & Handwerk $+s+z e n g$ \\
\hline 4 & Follbesprechung & Verfall $+s+$ datum \\
\hline 5 & Kanfpreis & Verkanf $+5+$ prets \\
\hline 6 & Rufname & Benuf $+s+n a m e$ \\
\hline 7 & Fahrizent & Abfabrt+s+zeit \\
\hline 8 & Schlagkwat & Vorschlag $+s+r e c h t$ \\
\hline 9 & Fangarm & Anfang $+s+$ gehalt \\
\hline
\end{tabular}

Tab. 2: Mono- und polymorphematische Erstglieder und Fugensetzung

\begin{tabular}{c|c|c|c|} 
Fugenelement & $\begin{array}{c}\text { Alle } \\
\text { Komposita }\end{array}$ & $\begin{array}{r}\text { Komposita mit morphologisch komplexen } \\
\text { Erstgliedern }\end{array}$ \\
$\begin{array}{c}\text { Erstglied = } \\
\text { Kompositum }\end{array}$ & Erstglied = Derivat \\
\hline Null & $58 \%$ & $66 \%$ & $29 \%$ \\
\hline$-5-$ & $25 \%$ & $27 \%$ & $67.5 \%$ \\
\hline
\end{tabular}

Tab. 3: Abhängigkeit der Fugenelemente Null und -s-

von der morphologischen Komplexität des Erstglieds (nach Kärschner 2003)

Diese Tatsache sowie ein Blick auf unser Korpus der Zweifelsfalle, das auBerdem ausweist, dass auffallig viele Fremdwort-Erstglieder in die Schwankungen involviert sind (s. Tabelle 1), hat uns in der Vermutung bestärkt, dass es phonologische Faktoren sein müssen, die das s-Fugenverhalten heute steuern. So haben wir in Nubling/Szczepaniak (2008) eine Korpusuntersuchung durchgefuhrt, indem wir anhand einer Zufallsauswahl 40 derivationell komplexe Erstglieder gewonnen haben und sämtiche Komposita mit diesen Erstgliedern auf ihr Fugenverhalten hin untersucht haben (Korpus: Cosmas II, Gesamtarchiv der geschriebenen Sprache mit uber I Millarde Wörtem). Etwa die Halfte dieser Erstglieder hatte unbetonte Präfxe, die andere Halfre betonte. Diese sind in Tabelle 4 aufgefuht. 


\begin{tabular}{|c|c|c|c|}
\hline \multicolumn{2}{|c|}{ Unbetonte Präfixe (21) } & \multicolumn{2}{|c|}{ Betonte Präfixe (19) } \\
\hline $\begin{array}{l}\text { Bedarft } \\
\text { Bedenkent } \\
\text { Befeht } \\
\text { Bestand+ } \\
\text { Bezinkt } \\
\text { Entgeltt } \\
\text { Entscheidt } \\
\text { Entsetzent+ } \\
\text { Entwurft } \\
\text { Bntzugt } \\
\text { Geburt+ }\end{array}$ & $\begin{array}{l}\text { Geduld } \\
\text { Gefugelt } \\
\text { Gelenkt } \\
\text { Gesangt+ } \\
\text { Verbrechent } \\
\text { Verbundt } \\
\text { Verdeckt } \\
\text { Verfallt } \\
\text { Verkant+ } \\
\text { Zerfall }\end{array}$ & $\begin{array}{l}\text { Anfahrtt } \\
\text { Anlangt } \\
\text { Anrechtt } \\
\text { Ansichtt } \\
\text { Aufsehent } \\
\text { Aursichtt } \\
\text { Aufstandt } \\
\text { Aufritt- } \\
\text { Aufuchtt } \\
\text { Uberfallt } \\
\text { Ubergangt }\end{array}$ & $\begin{array}{l}\text { Uberlebent } \\
\text { Ubermaßt } \\
\text { Uberschrift- } \\
\text { Ubersichtt } \\
\text { Unland. } \\
\text { Unzauscht } \\
\text { Umweltt } \\
\text { Unzugt }\end{array}$ \\
\hline
\end{tabular}

Tab. 4: Korpus der derivationell komplexen Erstglieder

Das Ergebnis, das Tabelle 5 enthäl, war eindeutig: Erstglieder mit unbetonten Präfixen werden zu $85 \%$ verfugt, solche mit betonten Präfixen nur zu 36\% (nach Tokens; die Untersuchung der Types erbrachte ähnliche Resultate).

\begin{tabular}{|c|c|c|}
\hline & \multicolumn{2}{|c|}{ Erstglieder mit ... } \\
\hline & unbetontem Präfix & betontem Präfix \\
\hline Tokens: & $\begin{array}{c}85 \% \\
\text { (von insg. } 495.887 \text { Komposita) }\end{array}$ & $\begin{array}{l}36 \% \\
\text { (rou, nom. } 324.503 \\
\text { Komposita) }\end{array}$ \\
\hline Types: & $\begin{array}{c}82 \% \\
\text { (von insg. 17.999 } \\
\text { Komposita) }\end{array}$ & 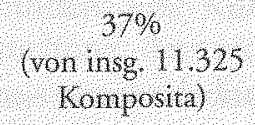 \\
\hline
\end{tabular}

Tab. 5; Das Fugenverhalten der derivationell komplexen Erstglieder

Damit haben die entscheidenden Kontrastpaare nicht $H o f+\varnothing+$ tor vs. Friedhof $+s+$ tor zu lauten, sondern Beruf $+s+$ wunsch vs. Anruf $+\varnothing+$ beantworter (neben Ruf $+\varnothing+$ mord) oder Verfall $+s+$ datum vs. Uberfall $+\varnothing+$ kommando (neben Fall $+\varnothing+$ beispiel). Selbstverständlich gibt es Ausreißer in beide Richnungen, wie die Zahlen oben ausweisen. Auch gibt es darunter einige Zweifelsfalle, z.B. Gewicht $(+s+$ ? $)$ heber ${ }^{8}$ und Antrag $(+s+$ ? $)$ formular aus Ta-

8 Dass Gewiht $(+5+$ ?) heber schwankt, liegt an seinem deverbalen Zweirglied. Diesen Gucenhemmenden Fakror, der vom Zweitglied ausgeht, vernachlässigen wir in diesem Beitrag. Thm wird an anderer Stelle nachgegangen. belle 1. Da wir es bei der $s$-Fuge mit einem in vollem Gang befindlichen Sprachwandel zu tun haben, ergeben sich keine harten Regeln, aber sehr deutliche Tendenzen: Das Fugenverhalten wird heute phonologisch gesteuert, und zwar in direkter Abhängigkeit von der phonologischen Wortqualität des Erstglieds. Je besser seine phonologische Wortqualität, desto weniger wird s-verfugt - und je schlechter seine phonologische Wortqualität, desto eher wird sverfugt. Dies illustriert Abbildung 1.

phonologische Wortqualitat:

gur

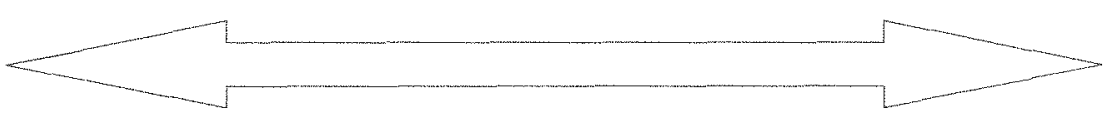

stark

Produktivitât der s- Fuge:

schwach

........viele Zweifelsfälle.............

Derivate mit unbetontem Präfix
oder mit nebenbetontem Suffix
Beruf + s+wunsch,
Berufung $+5+z$ usage.

Detivate mil betontem Prifix, Komposita:

Anruf O + beantworter

Weckufor onktion

Fremdwörter

Station $+s+$ äratin Respekt+s+person Präteritum + s? + schumd

Abb. 1: Die Abhängigkeit der s-Fugensetzung von der phonologischen Wortçualität des Erstglieds

Abbildung 1 hat auch die Fremdwörter integriert, die auffallend häufig s-verfugt werden, im Falle der Suffixe -ität und -ion sogar regelhaft, denn alle diese Wörter weichen maximal vom idealen phonologischen Wort des Deutschen $\mathrm{ab}$, das in einem Trochäus mit Reduktionssilbe besteht (Typ Mutter, Leute, Brunnen, Schliussel). Nur wenn solche Erstglieder deverbal, also mit Infinitiven homophon sind, werden sie uber des Fugen-s nominalisiert, d.h. hier steht die $s$-Fuge regelhaft: Lebents+mittel, Essen+s+ration, Wissen+s+durst (s, auch die Regel im Duden-Wörterbuch der sprachlichen 
Zwefelsfalle unter 2.1). Worter auf-ität und -ion weichen maximal rom phonologischen Wortideal ab: Sic enthalten weit mehr als zwei Silben, ja sogar in der Regel mehrere FüBe (mit unbetonten Silben, die Vollvokale enthalten), und statt einer fnalen Reduktionssilbe enthalten sie eine finale Vollonsibe (vgl, Internationalitats + kriterium, Integrationts+debatte). Dies gilt auch fur andere finabetonte Fremdwörter, vgl. Bibliothek+s+verwaltung, Instituts+versammlung, Dekanat+s+besetzung etc. wenngleich sich hierunter auch vicle Zweifelsfalle befinden, konkret die folgenden aus Tabelle 1: Abitur $(+5+$ ? feier, Seminar $(+5+$ ? $)$ arbeit, Referat $(+5+?)$ besprechung, Respekt $+s+$ ?) person. Auch für native Wortbildungen gilt obligatorische Verfugung, wenn sie auf voll-oder nebenbetonte Suffixe enden, daher die Regel, dass Wörter auf -heit, -(ig)keit, -schaft, -tum und -ung immer verfugt werden: Eimheits+brei, Geschwindigkeits+begrenzung, Bercischaft $+s+$ dienst, Eigentum $+s+w o h n u n g$, Einigung $+s+$ vertrag. Speziell dem Fugenverhalten bei Fremdwörtern sind wir in einem weiteren Aufsatz nachgegangen (Nubling/Szczepaniak 2009).

Selbst wenn die letzte Silbe unbetont ist, aber einen Vollvokal enthäl. wird of verfugt - und genau auf dieser Stufe befinden sich besonders vielo unserer Zweifelsfälle: Präteritum $(+s+?)$ schwund, Datum $(+s+?)$ angabe, Subjot $+s+?$ ) pronomen, aber auch verdunkelte Komposita sind prosodischphonologisch nicht weit davon enfernt wie z.B. Denkmal( $+5+3) p$ plege, Merkmall $+s+$ ?) analyse, Haushalt $(+s+$ ?) gerät. Der Zweifelsfall Interessen $(+s+$ ? vertretung ist durch seine Vokaltilgung schon dicht an einem Trochäus, doch enthalt er eine prätonische Vollsibe. Am wenigsten verfugt werden dem phonologischen Wortideal nahestehende Trochäen und Einsil-

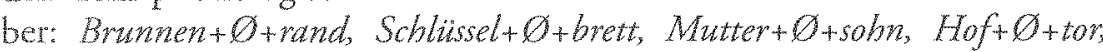
Tü $+D+$ schloss. Diese lange und fein abgestufte Skala zwischen schlechter und guter Wortqualität, die in Abbildung 1 nur angedeutet ist, wird detailliert in Nubling/Szczepaniak $(2008,2009)$ präsentiert und diskutiert.

Damit ist festzuhalten: Das Fugen-s reagiert auf die Qualiät des phonologischen Worts des Erstglieds und ist ein Indikator fur schlechte phonologische Wortqualität: Je größer die Distanz zum Trochäus, desto eher wird verfugt. Doch nicht nur das: Zentral ist die Tatsache, dass das Fugen-s immer eine Verstärkung des rechten Wortrands bewirkt, sei es, dass es den Rand nur erweitert und damit komplexer macht (Eigentum+stwohnung, Einigungtstvertrag, Religiontstunterricht), sei es, dass es gar extrasilbischen Status erlangt, indem es den abnehmenden Sonoritätsverlauf im Endrand konterkariert (Bereitschaff+s+diensts Stablitat+s+pakt, Geschwindigkeit $+s+b e g r e n z a n g)$. Damit "versiegel" ${ }^{\text {das }}$ Fugen-s das Erstglied. Dies enklärt auch, weshalb sich auffallig viele auf Plosiv endende Erstgliedkomposita (die grundsäzlich jeweils zwei phonologische Wörter bilden) unter den Zweifelsfallen befinden: Lehramt $(+s+$ ? kandidat, Schiffahn $(+5+3)$ unfall, Dreieck $(+s+?)$ wuch, auch Seehund $(+5+?)$ bank etc. Es ist kein Zufall, dass ausgerechnet das $-s$ diese Karriere gemacht hat und kein anderes Fugenelement.

2.5 Die typologische Perspektive:

Die s-Verfugung als Ausweis zunehmender Wortsprachlichkeit

Die s-Fuge hat ihre Domäne in der Wortbildung (Komposition) und dient hier als Grenzsignal. Damit liefert sie dem Hörer wichtige Informationen, sie bildet eine Dekodierungshilfe. Ungekehrt stellt die s-Fuge immer eine Ausspracheerschwernis dar, auch wenn schlichte Popularliteratur das Gegenteil annimme. Das heilst, auf der Sprecherseite verursacht die s-Fuge Kosten. Da die deutschen Sprecher jedoch komplexe Worränder gewohnt sind, fallt dieser weitere Komplexitätszuwachs kaum auf.

Damit ist ein Zusammenhang angesprochen, der ein Leimotiv der deutschen Sprachgeschichte seit mittelhochdeutscher Zeit bildet und erstmals von Szczepaniak (2007) beschrieben wurde: Das Deutsche hat sich typologisch grundlegend gewandelt. Es hat sich von althochdeutscher Sibensprachlichkeit zu (früh)neuhochdeutscher Worsprachlichkeir entwickelt. Exponiert und optimiert das Ahd. die Sprecheinheit Silbe, so exponiert das Nhd. die Informationseinheit Wort. Der Umbruch erfolgt im Mittelhochdeutschen und ist stark durch die Nebensilbenabschwächung bedingt, aber auch durch eine Reihe weiterer Faktoren, die sich hier nicht exhaustiv darstellen lassen (für einen Uberblick s. neben Szczepaniak (2007) auch Szczepaniak (2008; 2009, im Druck b). Nur stichwortartig seien einige diesbezuglich zentrale Unterschiede zwischen der ahd. Silbenund der nhd. Wortsprachlichkeit aufgefuhrt:

- Die ahd. Sibenstrukturen sind weitaus einfacher und dem CV-Ideal näher als im Nhd. (s. auch Werner 1978 zu einer Untersuchung der phonotaktischen Komplexitätszunahme des Endrands wortinaler Silben vom $M h d$. zum Nhd.). Epenthesen dienen im Ahd. der Herstellung von CV (ahd. burug ,Burg), während sie im (Frih-) Nhd. den rechten Wortrand verstärken (niomand Obstetc) und andere mor-

9 So lauter denn bei Bastian Sick der Ratschlag: „Dort, wo das Fugenz-s unaussprechich

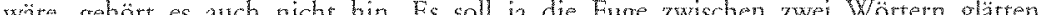
niche dieselbe zu in Zungention me

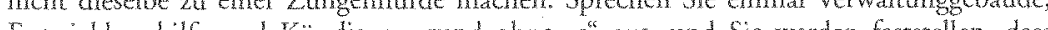
Entwicklughilfe cins es nicht nur blode kingt, sondem auch schweret zu artikuleren ist. Das Fugen-s wurde auch deshalb eingefigt, um das Wort leichter uber Zunge und Lippen zu bringen. Eine Aussprachehilfe, gewissermaken" (Sick 2004: 103). 
phologische Grenzen anzeigen (erwa systematisch die yor dem Suffix - hich, sofern auf [n] folgend: ordentich, namentich, versehentich). Da - lich noch heute ein eigenes phonologisches Wort bildet, handelt sich bei diesem $t$-Einschub um nichts anderes als ein Fugenelement.

- Auch der ahd. $i$-Umlaut (sowie andere Vokalharmonien) war sibenbezogen und erleichtert die Aussprache durch die artikulatorische Annaherung benachbarter Silbennuklei. Davon waren gleichermaßen die Fugenvokale ahd. Komposita betroffen, die noch keinerlei wortsegthentierende Funkrion hatten und leicht assimiliert (und auch elidiert) wurden (maggzogo statt maguzogo; klasougi (klascougi).

- Der (qualitative wie quantitative) Vokalismus war im Ahd. noch weitgehend symmetrisch, d.h. von der Akzentposition unabhängig, während im Nhd. eine Segregation besteht zwischen 18 Vollvokalen (einschl. Diphthongen) gegenüber zwei sog. Reduktionsvokalen ([o] und [e]), streng korrelierend mir Haupt- vs. Unbetontheit der betreffenden Silben.

- Phonetische und phonologische Prozesse waren im Ahd. silbenbezogen (s. etwa die 2. Lautverschiebung), während sie heute wortbezogen sind. Auch hat zum Nhd. hin eine Regulierung des phonologischen Wortes auf einen zweisilbigen Trochäus stattgefunden.

- Geminaten optimieren Silbengrenzen und galten noch für das Ahd.; sie sind heute abgebaut und durch ambisibische Konsonanten ersetzt worden, die schlechte Silbenkontakte bilden.

- Die kontinuierlichen Sonoritätsverläufe innerhalb der Silben werden zum Frihnhd. hin durch die Entstehung extrasilbischer Konsonanten an den Worträndern verschechtert (oft bedingt durch die Synkope) und mutieren damit zu einer wortpositionellen Information (vgl. Stadt $[\mathrm{t}-]$, Witz $[-\mathrm{ts}])$.

- Gegenwärtig entstehen in unbetonten Siben, ebenfalls synkopebedingt, massenhaft silbische Nasale und Liquide, die jenseits optimaler Sibenkerne stehen.

- Auch der weitere Konsonantismus verhält sich zunehmend wortbezogen, erkennbar ewa an der Aspiration anlautender Plosive oder der Auslautneutralisierung.

- Der sog. harte Vokaleinsatz, der Glottisverschlusslaut, hat im Ahd. noch nicht bestanden - er tut dies aber heute und markiert dabei den Wortanfangsrand. Manche silbensprachlichen Dialekte wie das Schweizerdeutsche haben ihn bis heute nicht ausgebildet (s, hierzu Nubling/Schrambke 2004). Wann er im Deutschen aufkommt, ist bis heute ungeklärt.
Genau in diesen Kontext fügt sich die Entstehung und vor allem Ausweitung des Fugen-s ein. Wie mehrfach gezeigt, verschlechtert es durch seinen extrasilbischen Status den Sonoritätsverlauf im Endrand, d.h. eine sibenoptimierende Funktion kommt ihm keinesfalls zu. Es dient nur der Anzeige schlecht zu erkennender Wörter, die von der idealen Wortphonologie zu stark abweichen. Diente es deren wortphonologischer GröBenoptimierung, so wäre die Nutzung der silbischen es-Fuge zu erwarten. Dies ist jedoch nicht eingetreten (die es-Fuge tritt nur sehr begrenzt auf und ist nicht produktiv). Ein solcher Effekt lässt sich durchaus fü das nächst häufige Fugenelement ausfindig machen, nämlich -(e)n, das tatsächlich komplementär distribuiert ist, indem es bereits bestehende Trochäen durch $+n+$ bewahrt (Pfanze+n+beet) und nicht bestehende in solche uberfuhrt (Fraw+en+sport); $-s$ als das produktivste und innovativste aller Fugenelemente hat diese Möglichkeit jedoch nicht genutzt (vgl. Wint+s+haus, Kind $+s+$ kopf, Schiff $+5+k a p i t a n)$. Es lassen nicht einmal Zweifelsfalle finden vom Typ *Referat + es + besprechung, ${ }^{*}$ Gewicht + es + heber oder * Krieg+es+fib hrung. Vielmehr hat Wegener (2006) festgestell, dass -s umso eher an Einsilber tritt, je mehr Extrasilbizitat es erzeugt: Einsilber, die auf Plosiv enden, werden deutlich ofter s-verfugt (Ont+s+tarif, Wints+haus) als solche, die auf Sonoranten enden (Wein $O+$ flasche). Damit dient das Fugen-s nicht nur als Indikator für schlechte phonologische Wörcer, vielmehr stellt es eine Ant Maßnahme zur Herstellung bzw. Verstäkung derselben dar. Das Fugen-s stärkt also den rechten Wortrand und exponiert furr den Hörer das Ende des (wegen seiner geringen Wortqualität schlecht zu erkennenden) Erstglieds.

\section{Fazit}

Ein aktueller und stark nachgefragter Zweifelsfall, die schwankende s-Verfugung, wurde als gegenwärtiger Vollzug sprachlichen Wandels identifiziert: Zahlreiche, vermutlich in die Hunderte gehende Komposita wechseln derzeit von der Null-zur s-Verfugung und generieren dadurch Zweifelsfalle $($ Seminar $+\varnothing+a r b e i t)$ Seminar+s+ambet $)$. Anhand einer Korpusrecherche haben wir gezeigt, dass es nicht die morphologische Komplexitat ist, die das derzeirige Fugenverhalten steuert, sondern die phonologische Komplexität: Je schlechter die phonologische Wortqualität des Erstglieds, d.h. je größer seine Distanz zum Trochäus mit Reduktionssibe, desto cher wird es verfugt. Heute operiert die s-Fuge im Mittelbereich, d.h. dort generiert sie die meisten Zweifelsfalle. Die besonders stark vom Trochäus mit Reduktionssibe abweichenden Wörter werden bereits obligatorisch sverfugt und verursachen daher kaum Zweifelsfalle. Dazu gehören va. die 
Dervate mit unbetonten Präfx (Gebrauch+s+anweisung). Derivate mit betontem Präfx sowie Komposita und zahlreiche Fremdwörte: erzeugen das Gros der heutigen Fugenschwankungen. Die generelle Tendenz geht zu vemehrter Komposita-Verfugung. Damit hat die s-Fuge einen langen Weg zuruckgelegt von einem (frühneuhochdeutschen) Flexionssuffix innerhalb eines syntaktischen Gefuges, das als Grenzmarker reanalysiert wurde und in die Wortbildung abwandert ist, um sich dort zu einem phonologischen Wortendrandverstätker zu entwickeln: Wie gezeigt, signalisiert das Fugen-s nicht nur schlechte Wortqualität, es trägt seinerseits zu hiher Erhohung bei. Aus typologischer Perspektive bestätigt die Existenz und weitere Ausdehnung dieses Fugenelements die prosodisch-phonologische Drift des Deutschen von einstmals einer Siben-zu heute einer Wortsprache.

Ohne die Kenntnis der Diachronie, dies sollte dieser Beitrag deutlich gemacht haben, wäre die gegenwärtige synchrone Fugenschwankung nicht zu verstehen.

\section{Literatur}

Andresen, Karl Gustaf (1923): Sprachgebrauch und Sprachrichtigkeit im Deutschen, Leipzig.

Augst. Gerhard (1975): Uber das Fugenmorphem bei Zusammensetzungen, in: Augst, Gerhard (Hg.): Untersuchungen zum Morpheminventar der deutschen Gegenwartssprache, Tubingen, 71-155.

Eisenberg, Peter (32006): Grundriss der deutschen Grammatik, Band 1: Das Wort, Stuttgart.

Fuhrop, Nanna (1996): Fugenelemente, in: Lang, Ewald/2ifonun, Gisela (Hg.) Deutsch - typologisch, Berlin, 525-550.

Fuhrop, Nanna (1998): Grenzfalle morphologischer Einheiten, Tubingen.

Gallmann, Peter (1998): Fugenmorpheme als Nichr-Kasus-Morpheme, in: Butt, Miriam/Fuhrhop, Nanna (Hg.): Variation und Stabilität in der Wortstruktur, Hildesheim, 177-190.

Henzen, Walter (1965): Deutsche Wortbildung, Tübingen.

Klein, Wolf Peter (2003): Sprachliche Zweifelstille als linguistischer Gegenstand. Zur Emfuhrung in ein vergessenes Thema der Sprachwissenschaft, in: Linguistik online $16,4,1-26$.

Klein, Wolf Peter (2009): Auf der Kippe? Zweifelsfalle als Herausforderung(en) tur Sprachwissenschaft und Sprachnormierung, in: Konopka, Marek/Strecker, Bruno (Tg.): Deutsche Grammatik - Regeln, Normen, Sprachgebrauch. IDS Jahrbuch 2008 , Berlin /New York, 141-165.

Nitta. Haruo (1987): Zur Forschung der, uneigendichen' Zusammensetzung im Frihneuhochdeutschen, in: Zeitschriff fur deutsche Philologie 106, 3, 400-416.

Kürshner, Sebastian (2003): Fugenelemente im Deutschen und Dänischen - eine Kontrastive Studie zu einem Grenzfall der Morphologie, Freiburg (Internet: www. freidok uni-freburg.de/vollexte/12560.

Matthias, Theodor (1906): Sprachleben und Sprachschaden, Leipzig.
Nübling, Danaris/Schrambke, Renate (2004): Silben- versus akzentsprachliche Züge in den germanischen Sprachen und im Alemannischen, in: Glaser, Elvira et al. (Hg.): Alemannisch im Sprachvergleich, Wiesbaden, 281-320.

Nuibling, Damaris/Szczepaniak, Renata (2008): On the way from morphology to phonology: German linking elements and the role of the phonological word, in: Morphology $18,1-25$.

Nubling, Damaris/Szczepaniak, Renata (2009): Religion+s+freiheit, Stabilitüt+s+togkt und Subjekt $(+s+$ )-pronomen: Fugendemente als Marker phonologischer Wortgrenzen, in: Muller, Peter Otro (Hg): Studien zur Fremdwortbildung, Hildesheim, $195-222$.

Orner, Lorelies et al. (1991): Deutsche Worbildung. Typen und Tendenzen in der Gegenwartssprache, Bd. 4: Substantivkomposita, Berlin/New York.

Ravlov, Vladimir M. (1972): Die substantivische Zusammensetzung in Deutschen als syntaktisches Problem, München.

Pavlov, Vladimir (1983): Zur Ausbildung der Norm der deutschen Literatursprache im Bereich der Wortbildung (1470-1730). Von der Wortgruppe zur substantivischen Zusammensetzung, Berin.

Sattler, Lutz (1992): Zur Verwendung von Wortgruppen mit adjektivischem Atribut und Komposita in der deutschen Literatursprache (1570-1730), in: Joachim Schildt (Hg.): Aspekte des Sprachwandels in der deutschen Literatursprache 1570-1730, Berlin, 227-271.

Sick, Bastian (2004): Der Dativ ist dem Genitiv sein Tod, Koln.

Solms, Hans-Joachim (1999): Der Gebratch uneigentlicher Substantivkomposita im Mittel- und Früneuhochdeutschen als Indikator kulureller Veränderungen, in: Gardt, Andreas et al. (Hg.): Sprachgeschichte als Kulturgeschichte, Berlin/New York, 225-246.

Steche, Theodor (1925): Neue Wege zum reinen Deutsch, Breslau.

Steche, Theodor (1927): Die neuhochdeursche Wortbiegung unter besonderer Berücksichtigung der Sprachentwicklung im 19. Jahthundert, Breslau.

Szczepaniak, Renata (2007): Der phonologisch-typologische Wandel des Deutschen von einer Silben-zu einer Wortsprache, Berlin /New Yodk.

Szczepaniak, Renata (2008): Phonologischer Wandel, in: Nubling, Damaris et al. (Hg): Historische Sprachwissenschaft des Deutschen. Eine Einfuhrung in die Prinzipien des Sprachwandels, Tübingen, 11-42.

Szczepaniak, Renata (2009): Wortsprachliches Deutsch und silbensprachliches Spanisch. Ein phonologisch-typologischer Vergleich, in: Estudios flológicos alemanes, 17 , $251-267$.

Szczepaniak, Reriata (im Druck a): Whihrend des Flug(e) des Ausflug(e)s? German Short and Long Genitive Endings between Norm and Variation, erscheint in: Lenz, AIexandra/Plewina, Albrecht: Grammar Between Norm and Variation (Studies in Linguistic Variation, Berlin/New York.

Szczepaniak, Renata (im Druck b): Phonologisch-typologischer Wandel des Deutschen und des Luxemburgischen im Kontrast, erscheint in: Dammel, Antie/Kürchner: Sebastian/Nübling, Darnaris (Hg.): Kontrastive gemanistische Linguistik, Hildesheim.

Wegera, Klaus-Perer/Plett, Heinz-Peter (2000): Wortbildung des Frihnewhochdeutschen, in: Besch, Werner et al. (Hg): Sprachgeschichte, Bd. 2. Berlin/New York, $1594-1605$. 
Wencer Ormar (1978): Schwa-Schwund und Phonotaktik im Deutschen, in: Birnbaum, Henrik et al. (Hg.), Studia Linguistica A. V. fllio Issatschenko a Collegis Amicisque oblata. Lisse, $471-486$.

Wilmanns, Whelm (1899): Deutsche Grammatik, Bd. 2: Wortbildung, Straßburg.

Wusmann, Gustav (1891): Allerhand Sprachdummeiten. Kleine deutsche Grammatik des Zweifelhaften, des Falschen und des Häßlichen, Leiprig.

Peter Entst (Wien/Leipzig)

\section{Zum Problem „Mündlichkeit - Schriftichkeit" in der deutschen Sprachgeschichte}

Ein Vorschlag für ein neues Kommunikationsmodell in Diachronie und Synchronie

\section{Ausgangslage}

Für einige linguistische Teildisziplinen hat sich die Doktrin vom Primat der gesprochenen Sprache bekanntlich nicht durchgesetzt. Dazu gehören vor allem Orthografie, Grammatik und Sprachgeschichtsschreibung. Die Historische Sprachwissenschaft gehorcht dabei der Not, dass für den überwiegenden Teil der Sprachgeschichte nur schriftliche Zeugnisse zur Verfugung stehen. Aber auch seit dem Beginn des 20. Jahrhunderts, als erstmals Gesprochenes in einer einigermaßen akzeptablen Qualität konserviert werden kann und systematisch gesammelt wird, ist eine deutliche Konzentration auf schriftliche Quellen zu erkennen. Dies hängt nicht nur mit Forschungstraditionen zusammen, sondern auch mit dem schwer wiegenden methodologischen Problem, wie gesprochene Sprache als "langue" einzuordnen ist. Letztlich fehlt aber auch ein zeitgemäßes Kommunikationsmodell, auf dessen Basis die mündlichen und schriftlichen Kommunikationsvorgänge zuallererst beschrieben werden müssten.

Im Jahr 1900 wurde das Phonogrammarchiv der Kaiserlichen Akademie der Wissenschaften zu When, weltweit die erste Einrichtung dieser Art, gegrindet. Aus ihren Beständen stammen die folgenden beiden Mitschriften von Redeausschnitten, die anlässlich der Einfihrung des allgemeinen und gleichen Wahlrechts 1906 im Wiener Reichsrat gehalten worden sind. 

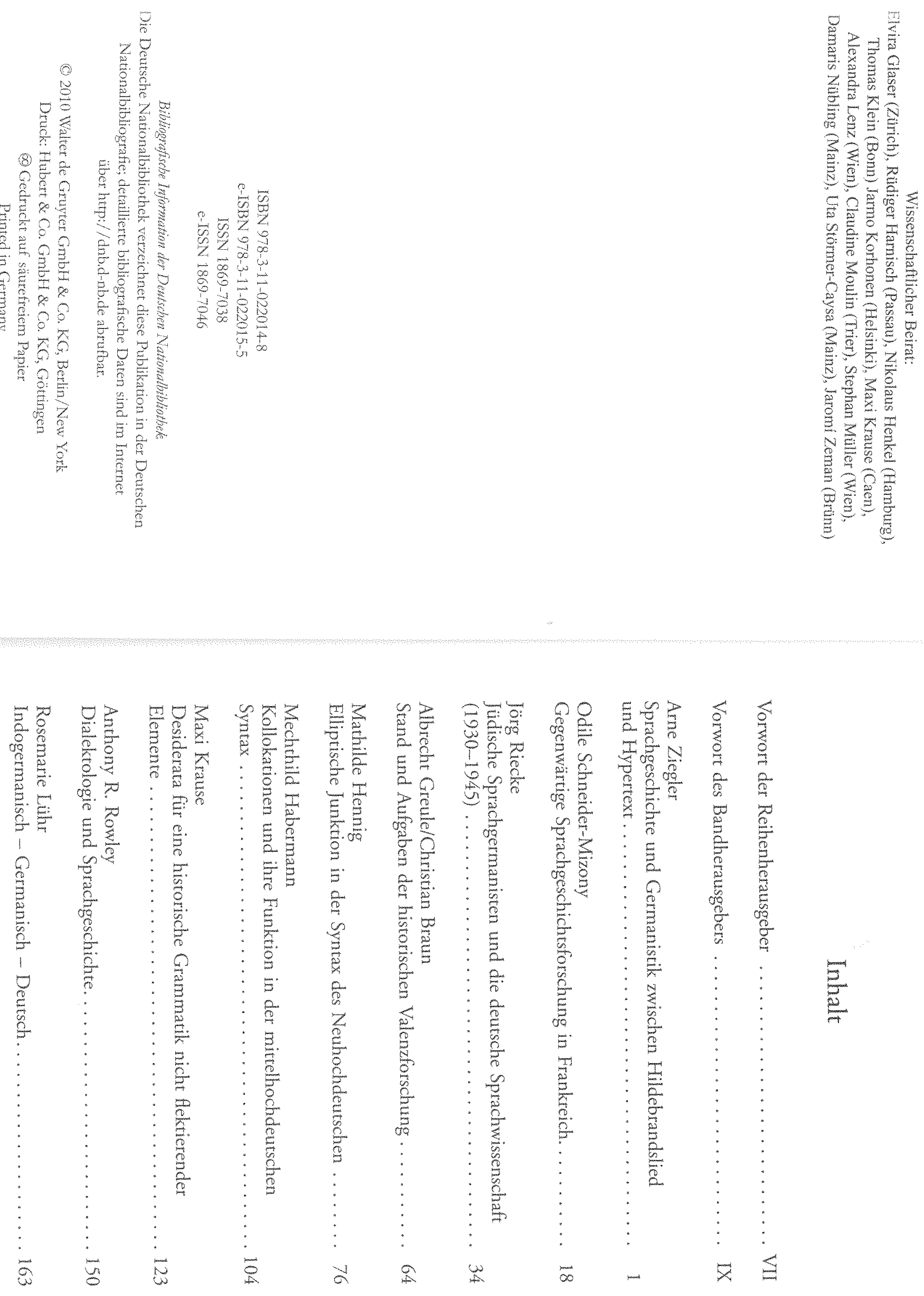